\title{
Endodontic and Periodontal Management of an Apical Fenestration: A
} Case Report

\author{
Ayari Ghada $^{12^{*}}$, Nasri Wafa ${ }^{3}$, Bagga Sana ${ }^{1},{ }^{2}$, Hamdane Khaireddine $^{3}$, Ben Hadj Khalifa Aymen ${ }^{1}$, Sahtout Souad ${ }^{1}{ }^{2}$
}

${ }^{1}$ Biological and Clinical Dento-Facial Approach Laboratory (ABCDF) LR12ES10, Faculty of Dental Medicine, University of Monastir, Monastir, Tunisia

${ }^{2}$ Department of Endodontics, Dental Clinic of Monastir, Faculty of Dental Medicine, University of Monastir, Tunisia

${ }^{3}$ Department of Periodontology, Dental Clinic of Monastir, Faculty of Dental Medicine, University of Monastir, Tunisia

DOI: $10.36347 /$ sjmcr.2021.v09i03.002 $\quad$ | Received: 17.02.2021 | Accepted: 28.02.2021 | Published: 04.03.2021

*Corresponding author: Ayari Ghada

This study reports the case of a 52-year-old woman in good state of health (ASA) showed up with pain on biting, the gutta percha was visible through the alveolar mucosa exceeding the apex of the first upper right premolar two weeks after root canal treatment and the healing of a sinus tract. The CBCT exam revealed a buccal malposition of the tooth and the apical third of the buccal root perforated the alveolar buccal plate. The treatment rationale is presented and compared with other therapeutic options described in the literature. A root end resection in association with a bone filling from the adjacent site were performed to preserve the tooth. The evolution was favorable after surgery. The therapeutic approach gave satisfactory results.

Keywords: Periodontal access flap, bone fenestration, endodontic treatment, root end resection, endodontic surgery. Copyright $(\mathcal{C}) 2021$ The Author(s): This is an open-access article distributed under the terms of the Creative Commons Attribution 4.0 International License (CC BY-NC 4.0) which permits unrestricted use, distribution, and reproduction in any medium for non-commercial use provided the original author and source are credited.

\section{INTRODUCTION}

Two commonly encountered alveolar defects are dehiscences and fenestrations. Dehiscence is a deficiency of the alveolar bone margin. The root surface is covered only by the periosteum and the overlying gingiva. While a fenestration, is a circumscribed defect of the alveolar radicular bone that does not involve the alveolar margin [1].

The fenestrations are most often seen on the mesiobuccal root of the maxillary first molar and the canine teeth. Some authors also consider heavy occlusal forces as an etiologic factor for the development of fenestrations [2], or aggravating ones [3].

The objective of this case report was to describe a treatment modality used to manage an apical alveolar fenestration placed on the right first maxillary premolar buccal root.

\section{Case Report}

\section{Patient evaluation}

A 53-year-old woman showed up to the department of endodontics in faculty of dentistry of Monastir for the treatment of the tooth number 14 with defective amalgam restoration. The patient was in good state of health (ASA), extra oral examination did not reveal any pathologic signs, the tooth number 14 presented an old defective amalgam restoration (figure 1) and a sinus tract in the buccal mycosis, a diagnosis of an asymptomatic apical abscess was posed and an endodontic treatment was performed.

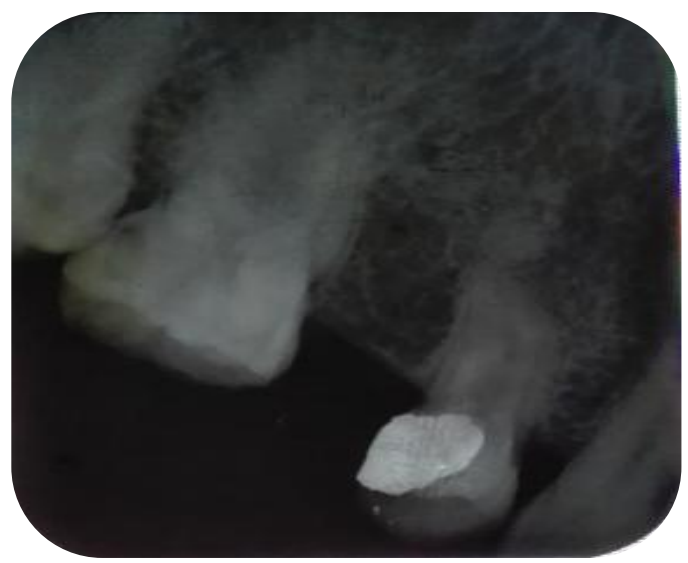

Fig-1: preoperative radiograph. First consultation

Two weeks later the patient presented with pain on biting, the healing of the buccal mycosis 
revealed that the gutta percha was visible through the gingiva (figure2 A).

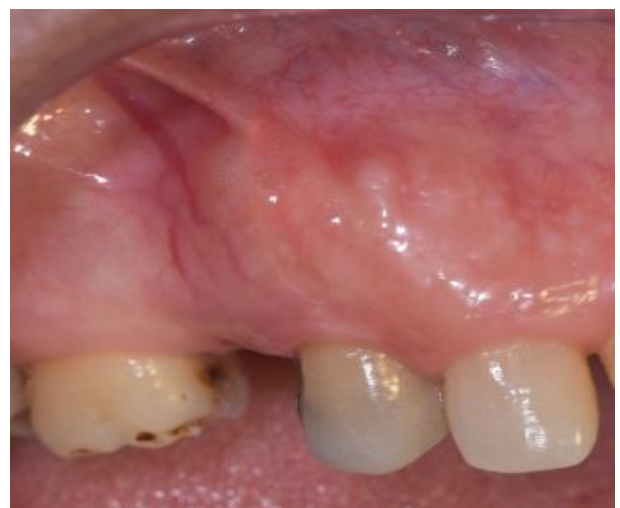

Fig-2A: initial clinical situation. The gutta percha visible through the alveolar mucosa

The examination at the control appointment revealed that other teeth in the maxillary area began to present a fenestration which can help to determine the etiologic factor of this osseous defect (figure 2B). In fact, the patient underwent the extraction of the left and right maxillary canines erupted with ectopic position at an early age which caused the mesial migration and rotation of teeth 14 and 24 and this can explain the buccal malposition.

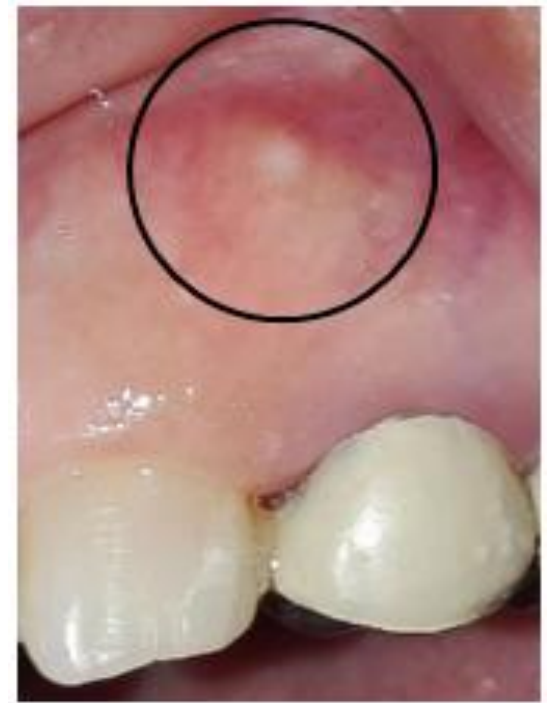

Fig-2B: clinical photograph of the tooth number 24

\section{Treatment \\ Preoperative examination}

Before deciding on the treatment plan, conebeam assessment of the periapical lesion was requested. The CBCT examination revealed a thin alveolar buccal plate, fenestrated in the apical third, buccal root protrusion and an over obturation of 2 millimeters in the same root (figure 3).
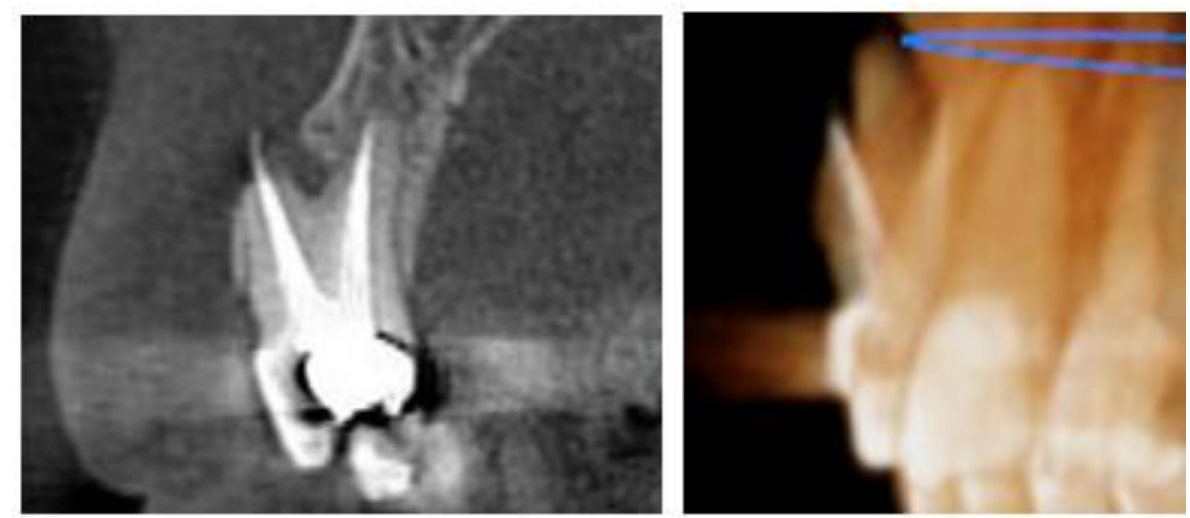

Fig-3: a coronal section (left) and a 3D reconstitution (right) of the tooth 14

For this purpose, Yoshioka $\mathrm{T}$ et al. classified periapical lesions according to the characteristics of the bone defect revealed by CBCT. They identified the fenestrations as "type V lesions" characterized by apical root protrusion of the buccal bone surface with a bony defect involving the buccal cortical plate. In cases where the protrusion is relatively small, such as type V subcategory 1 (protrusion of the apical foramen only) and subcategory 2 (the protrusion of the apical third of the root), surgical exposure of the apex and its remodeling within the surrounding bone tissue are the treatment of choice [4].

\section{Operative time}

The design of the flap is of great interest in endodontic and periodontal surgery. It affects access, visibility, respect of anatomical structures, and postoperative sequelae on the periodontium [5].

After performing disinfection and anesthesia, a triangular flap was made. It includes a horizontal incision, first intrasulcular and then crestal at the level of the adjacent edentulous ridge to collect autogenous bone later, associated with a mesial vertical relieving incision. The intrasulcular incision was limited to the affected tooth. The vertical relieving incision was performed on healthy bone at a distance from the periapical lesion and from the midway point between 
the gingival zenith and the top of the papilla to preserve it.

After drawing incisions, a full thickness flap was carefully elevated to avoid damage to fragile soft tissues, especially at the damaged area. The elevation should begin with the vertical relieving incision at the attached gingiva [5, 6]. For the interdental papilla a thinner periosteal elevator should be used to avoid trauma (figure 4A).

Healthy tissue removal was not necessary. Curettage of the granulation tissue was sufficient to facilitate access to the apex. Subsequently, the filling material protruding from the apex was removed (figure 4B).

After the apical resection (figure 4C), rigorous hemostasis was performed to allow retro-filling. This step is of great importance, especially for the placement of the filling material. The retrograde cavity was carried out using a gooseneck bur and filled with a rapid setting reinforced eugenate IRM® (figure 4D).
To fill the bone defect, autogenous bone chips from the adjacent edentulous ridge was used. In addition to their osteogenic role, they will serve as a mechanical support for the overlying tissues (figure 4E).

Finally, the flap was replaced and "o" stitches were made, first at the horizontal incision and then at the vertical relieving incision (figure 4F).

Postoperative advice was communicated to the patient. An analgesic and an antiseptic mouthwash were prescribed.

The coronal restoration was performed with a sandwich technique combining glass ionomer cement and composite resin, the prosthetic treatment was discussed and could not be performed because of the increasing of the ratio between radiological crown and radiological root.
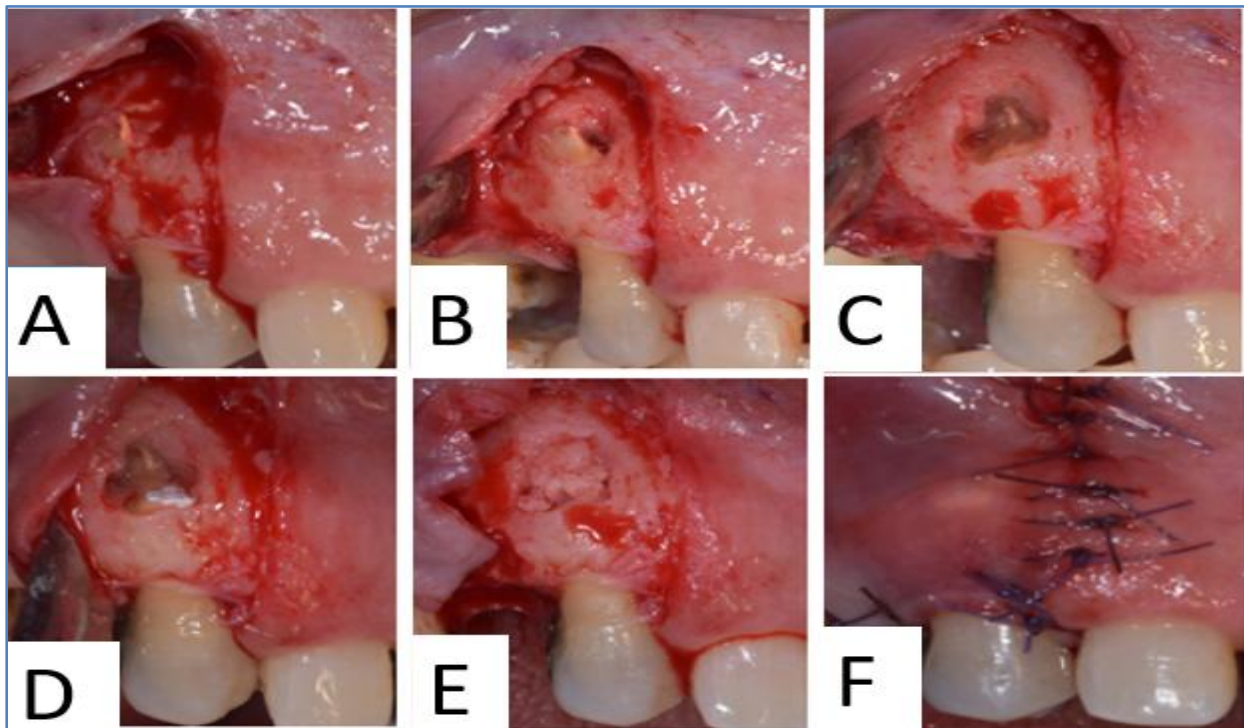

Fig-4: A- mucoperiosteal flap rising. B- Curettage of the periapical area. C- Root end resection and remodeling of the apex. DPreparation of the canal and obturation with IRM®. E- Bone filling of the periapical area. F- Assembly of the flap with nonabsorbable suture

\section{RESULTS}

The patient presented 7 days postoperatively for the removal of the stitches, mucosal healing was satisfactory.

\section{Follow up}

Six months after the intervention, patient didn't present any symptom, the mucosa was completely healed and the region was in perfect good shape. Periapical radiography revealed well performed endodontic treatment, the healing and remodeling of the apical area.

The patient was seen one year and two years after surgery, a clinical and radiographic examination revealed a satisfactory healing of the bone and mucosa with no recidivism (figure 5). 

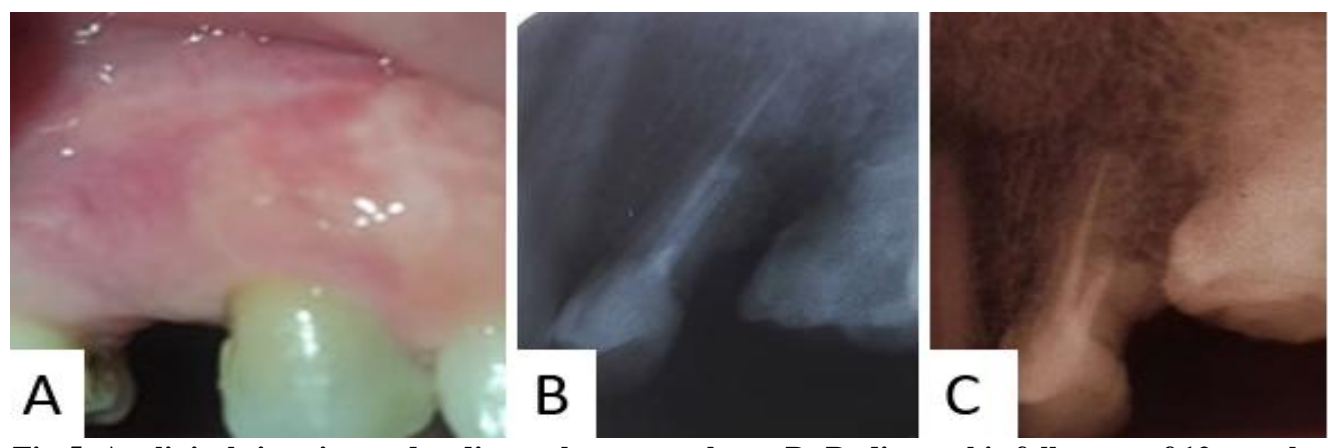

Fig-5: A- clinical situation and radiograph one year later. B- Radiographic follow up of 12 months. C- Radiographic follow up of 24 months

\section{DISCUSSION}

The defect in the osseous coverage of the dental root may either have been fenestration or dehiscence. They occur almost exclusively on the buccal surfaces of the alveolar bone [7].

The predisposing factors for these defects can be age, tooth malposition, Root prominences, tooth/jaw ratio, orthodontic tooth movement, trauma and strong occlusal forces. The fenestrations are most often seen on the mesiobuccal root of the maxillary first molar and the canine teeth. Some authors also consider heavy occlusal forces as an etiologic factor for the development of fenestrations [2], or aggravating ones.

Fenestrations and dehiscences are symptom free but can raise a flare up after an endodontic treatment because of the irritation of the gingiva by the exceeding of filling material or overinstrumentation, this pain is perceived mainly on mastication or apical palpation and may increase the prevalence of complications, although considered no pathological conditions, they also may complicate endodontic treatment, periodontal and periapical surgical procedures or require changes in implant placement protocols [3]

The evaluation by cone-beam computed tomography (CBCT) of the periapical lesion is recommended in this situation, as CBCT can reveal defects of the cancellous bone and cortical bone separately [8].

The treatment plan purpose was to generate an environment that would promote periapical healing, to repair hard tissue defect with respect to fenestration and to preserve the endodontic treatment from bacterial invasion by using a material with more improved capacity of sealing and adherence.

The clinical treatment consists of raising a flap; trimming the excess filling and remodeling of the root in such a way as to reposition it in the bony housing and at the same time improve accessibility and visibility around the apex for curettage. The bone may then repair itself and the soft tissue will heal. In the case presented herein, a bone from the adjacent site was used, so as to promote bone growth if possible. Even if bone doesn't form, the soft tissue will heal.

Periradicular surgery can be considered as a predictable technique for the treatment of teeth that have suffered endodontic failure. Von Arx et al. reported that its success rate is $83.8 \%$ at one year postoperatively [9].

In a systematic review, Torabinejad et al. concluded that it offered a greater initial success rate than non-surgical techniques (endodontic retreatment). On the other hand, its long-term results have been less favorable [10]

The use of isolated materials such a membrane or bone graft has not been well studied. In contrast, a number of studies of guided tissue regeneration in periapical surgery have combined the two. A systematic review by Sánchez-Torres et al. has reported that simultaneous use of membranes and bone grafts shows more predictable results than isolated techniques. This same study showed that the benefit of guided tissue regeneration is more significant for lesions with 3 walls than those with 4 walls [11].

In a review of the literature published in 2011 Von Arx et al. have shown that the use of guided tissue regeneration in periapical surgery for the treatment of 4 wall bone defects has minimal or no benefits. This is because the risk of soft tissue proliferation in the defect is low and bone neoformation may occur [12].

The root end resection was performed carefully to eliminate the minimum amount of tissue to preserve the tooth implantation and prevent mobility.

The apex remodeling and retrograde filling are performed to ensure an optimum apical sealing preventing bacterial invasion and subsequently an endodontic infection.

The rapid setting eugenate was used for the following: easier manipulation, a great humidity tolerance and low cost in comparison to other indicated materials. 
A global management plan was established to treat other teeth with fenestration, recommendations were proposed to avoid overinstrumentation and overfilling: using apex locator and radiographic evaluation can be insufficient to determine the working length, an apical filling performed during surgery was proposed to determine clinically the apical foramina and perform the apical resection and the retro filling at the same time.

Periodontal pathology associated with endodontic compromised treatment necessitates institution of restorative, endodontic and periodontal therapies in combination to achieve the best treatment outcomes. Collective treatment rendered in this case which included endodontic therapy and apical root coverage prevented bacterial colonization and penetration in both retrograde and orthograde directions thereby providing an environment favorable for healing and repair and for the maintain of the oral hygiene. Attachment of periodontal ligament with repair of fenestration and periapical healing could be appreciated both clinically and radiographically [8]

Radiological and clinical follow up is necessary to control endodontic complications and to intervene in case of recidivism or endodontic lesion; a therapeutic procedure was planned for the treatment of the other concerned teeth.

\section{CONCLUSION}

Although infrequent, the protrusion of the apical area of a dental root through the cortical requires a comprehensive approach by the practitioner. The therapeutic protocol gave satisfactory results in this clinical case.

\section{REFERENCES}

1. Edel A. Alveolar bone fenestrations and dehiscences in dry Bedouin jaws. Journal of Clinical Periodontology. 1981 Dec;8(6):491-9.
2. Agarwal V, Fenestration and dehiscence in a nonvital tooth - a case report, Indian J Neonat Med Res. 2010, 4(4):2971- 2973.

3. Boucher Y, Sobel M, Sauveur G, Persistent pain related to root canal filling and apical fenestration: a case report, J Endod. 2000, 26(4):242-244

4. Yoshioka T, Kikuchi I, Adorno CG, Suda H, Periapical bone defects of root filled teeth with persistent lesions evaluated by cone-beam computed tomography, Int Endod J. 2011, 44(3):245-252.

5. Gutmann JL, Harrison JW. Posterior endodontic surgery: anatomical considerations and clinical techniques. International Endodontic Journal. 1985 Jan;18(1):8-34.

6. Regan JD, Witherspoon DE, FoyleDeborahm. Surgical repair of root and tooth perforations. Endodontic Topics. 2005;11(1):152-178.

7. Spasser HF, Wendt R. A cause for recalcitrant post endodontic pain. NY State Dent J. 1973; 39:25-6.

8. Sarang Sharma, Dhirendra Srivastava, Vishal Sood, Priya Yadav, Endodontic and periodontal management of a severely affected maxillary lateral incisor having combined mucosal fenestration and palatogingival groove $\mathbf{J}$ Indian Soc Periodontol. 2015 May-Jun; 19(3): 348-351.

9. von Arx T, Jensen SS, Hänni S. Clinical and radiographic assessment of various predictors for healing outcome 1 year after periapical surgery. Journal of endodontics. 2007 Feb 1;33(2):123-8.

10. Torabinejad M, Corr R, Handysides R, Shabahang S. Outcomes of Nonsurgical Retreatment and Endodontic Surgery: A Systematic Review. Journal of Endodontics. 2009;35(7):930-937. doi:10.1016/j.joen.2009.04.023

11. Sánchez-Torres A, Sánchez-Garcés MÁ, GayEscoda C. Materials and prognostic factors of bone regeneration in periapical surgery: A systematic review. Medicina oral, patologia oral y cirugia bucal. 2014 Jul;19(4):e419.

12. von Arx T, AlSaeed M. The use of regenerative techniques in apical surgery: a literature review. The Saudi Dental Journal. 2011 Jul 1;23(3):113-27. 